\title{
Iron Deficiency Anaemia in Pregnancy: Diagnosis, Prevention and Treatment
}

\author{
Palihawadana TS ${ }^{1}$, Goonewardene $\mathrm{IMR}^{2}$, Motha $\mathrm{MBC}^{3}$, Williams HSA ${ }^{4}$
}

\section{INTRODUCTION}

Iron deficiency (ID)is the commonest nutritional deficiency among pregnant women worldwide. ID can manifest in a wide spectrum, with iron deficiency anaemia (IDA) being the most severe form. The WHO estimates IDA to affect approximately $42 \%$ of pregnant women and it has been found to be commoner in the non-industrialised countries. More than 56 million women worldwide are estimated to have IDA, with approximately 32 million of them being from Asia. IDA in Sri Lanka was estimated to be around $29 \%{ }^{1}$.Although a more recent study suggests this to be approximately $16.7 \%{ }^{2}$, as only 228 pregnant women were included in this latter study, the results should be interpreted with caution, until more reliable data are obtained. Other small scale studies done in the Western Province and Anuradhapura have also reported rates of $18 \%$ and $14 \%$ respectively ${ }^{3,4}$. A reliable estimate of the prevalence of IDA in Sri Lanka is needed, as this will enable a proper evaluation of its public health impact and significance (Table $1)^{5}$, which in turn is a pre requisite for the implementation of appropriate remedial, iron supplementation programs.

\footnotetext{
1 Lecturer, Department of Obstetrics \& Gynaecology, University of Kelaniya

2 Senior Professor \& Chair, Department of Obstetrics \& Gynaecology, University of Ruhuna

3 Senior Lecturer \& Consultant Physician, Department of Obstetrics \& Gynaecology, University of Kelaniya

4 Senior Lecturer \& Consultant Haematologist, Department of Pathology, University of Kelaniya
}

Correspondence: Dr. T. S. Palihawadana

E-mail: thilipali@kln.ac.lk

Competing interests: None

\begin{tabular}{|lc|}
\hline $\begin{array}{c}\text { Table 1: Classification of public health significance of anaemia in populations on } \\
\text { the basis of prevalence estimated from blood levels of haemoglobin }\end{array}$ \\
\hline Category of public health significance & Prevalence of Anaemia \% \\
\hline Severe & 40 or higher \\
Moderate & $20.0-39.0$ \\
Mild & $5.0-19.9$ \\
Normal & 4.9 or lower \\
\hline
\end{tabular}

In a country like Sri Lanka where wide variations are noted in socioeconomic status as well as in diet, the prevalence could show variations in different geographical and demographic subgroups. Since IDA is a major contributory factor for maternal morbidity as well as mortality, the national program caring for the antenatal women should be capable of providing satisfactory care. However, in a recent study done in Colombo, community based antenatal clinics were found to have major shortcomings in the services with regard to quality of care in detecting and treating IDA, with over $60 \%$ of clinics lacking a satisfactory test for detection of a low haemoglobin concentration $(\mathrm{Hb})$, and only $4 \%$ providing any education to the pregnant women when providing the iron supplementation ${ }^{4}$.

\section{DIAGNOSIS OF IRON DEFICIENCY ANAEMIA}

IDA is defined as a reduction of $\mathrm{Hb}$ below a certain threshold, due to ineffective erythropoiesis resulting from ID. Various threshold levels have been suggested for different situations. During a normal pregnancy, due to the expansion of plasma volume exceeding the increased production of erythrocytes up to the second trimester, the $\mathrm{Hb}$ starts decreasing in the first trimester, reaching its lowest point in the second trimester (up to a maximum decrease of approximately $0.5 \mathrm{~g} / \mathrm{dl}$ ), and begins to rise again in the third trimester ${ }^{5}$. Therefore some recommend an allowance for this reduction, in the diagnosis of anaemia during pregnancy ${ }^{6}$. However, a cut off level of $11 \mathrm{~g} / \mathrm{dl}$ at sea level, throughout pregnancy, is recommended by the $\mathrm{WHO}^{5}$. This will enable comparison of prevalence and effects of IDA in different communities, regions and countries. The only adjustments recommended to the cut off level of $11 \mathrm{~g} / \mathrm{dl}$ at sea level are: reductions ranging from $0.2 \mathrm{~g} / \mathrm{dl}$ at altitudes of $>$ 1000 metres to a maximum reduction of $4.5 \mathrm{~g} / \mathrm{dl}$ at altitudes of $>4500$ metres, and a reduction of $0.3 \mathrm{~g} / \mathrm{dl}$ for smokers up to a maximum reduction of $0.7 \mathrm{~g} /$ dl for those who smoke more than 20 cigarettes per day ${ }^{5}$.

Assessment of haemoglobin should be by a reliable method such as cyanmethemoglobin method in a laboratory setting, or using a portable system such as a HaemoCue ${ }^{\circledR}$ system which uses the same method. Assessment of haematocrit is sometimes used in field surveys to identify prevalence of anaemia, but its accuracy in quantifying anaemia is not adequate for it to be used in clinical practice.

Screening of all pregnant women for anaemia is recommended at the booking visit as well as in the early third trimester. Women with multiple gestations, teenage mothers and those with reduced inter pregnancy intervals are at a higher risk of IDA.

In the presence of a low $\mathrm{Hb}$, haematological indices obtained from a full blood count combined with a 
blood picture can strongly suggest a diagnosis of IDA. Although a mean corpuscular volume $(\mathrm{MCV})$ of $<80 \mathrm{fl}$, a mean corpuscular haemoglobin concentration (MCHC) of $<30 \%$ and a mean corpuscular haemoglobin $(\mathrm{MCH})$ of $<30 \mu \mathrm{g}$ are considered to indicate IDA, haematological indices are known to be altered during pregnancy ${ }^{7,8}$. Furthermore, a blood picture will characteristically show microcytic hypochromic red blood cells with anisopoikilocytosis and pencil shaped cells in IDA. In the presence of other nutritional deficiencies such as folate deficiency, which is common during pregnancy, a dimorphic blood picture with macrocytic red blood cells and relatively normal haematological indices may be found. A serum ferritin (SF) of $<12 \mu \mathrm{g} / 1$ is frequently used to diagnose ID, but this too may be elevated to $>20 \mu \mathrm{g} / \mathrm{L}$ in the presence of any chronic inflammatory condition, thereby masking underlying ID. It should also be appreciated that IDA is only a part of the iceberg of ID. It has been estimated that the prevalence of ID may be approximately twice that of $\mathrm{IDA}^{9}$. Therefore $\mathrm{Hb}$ per se is a poor indicator of iron deficiency ${ }^{10}$. A SF level of $<30 \mu \mathrm{g} / 1$ is considered clinically significant, since it is a good indicator of depleted iron stores, which can lead to functional impairment at tissue level ${ }^{9}$, and the woman is very likely to develop IDA if not treated ${ }^{8}$. Serum transferrin receptor measurements are considered reliable tests to diagnose ID, but are seldom used in clinical practice due to high cost. Assessment by staining a sample of bone marrow, which is considered the gold standard test for iron stores, is not practical due to its invasiveness. Other tests of iron status are considered less reliable or have limited value in clinical practice.

A trial of therapeutic oral iron up to two weeks can be a useful diagnostic tool when IDA is suspected ${ }^{6}$. A significant rise in the haemoglobin level would often confirm the diagnosis and will avoid the need for any further expensive testing. This is a very useful intervention since it can even be undertaken at the community setting. However, it should be noted that certain limitations do exist in the above diagnostic work up. Haemoblobinopathies such as thalassemia can also give rise to similar findings on haematological indices, and iron deficiency can also co-exist with such haemoglobinopathies.

Further investigations for assessment of iron stores and other abnormalities such as haemoglobinopathies should be undertaken in women who fail to show a satisfactory response to an oral iron trial and those with malabsoption syndromes.

\section{PREVENTION OF IRON DEFICIENCY ANAEMIA IN PREGNANCY}

Routine, daily, antenatal oral iron supplementation has been in practice for many decades. Although its effectiveness in correcting ID and IDA has been proven, the need for such supplementation and the beneficial effects on pregnancy outcomes in certain populations has been questioned in recent times. Many industrialised countries including UK have advised against universal supplementation in their clinical guidelines $^{6}$. However, routine, daily, antenatal oral iron supplementation has been shown to be associated with a reduction of low birth weight, prematurity and maternal ID and IDA at term ${ }^{11}$. Furthermore, correcting ID and IDA in the mother will reduce the risk of maternal morbidity and mortality due to a combination of anaemia with obstetric haemorrhage and sepsis as well as possible longterm adverse effects in the newborn ${ }^{12-15}$. Sub-optimal iron supplementation has been proposed as a factor for needing blood transfusion in the postpartum period $^{16}$.

The WHO recommends a policy of routine, daily, antenatal oral iron supplementation based on the estimated prevalence of anaemia. In settings with a high prevalence of anaemia $(>40 \%)$ a higher supplementation dose of $60 \mathrm{mg}$ of elemental iron per day is recommended, while a lower dose of $30 \mathrm{mg}$ per day is recommended in settings with a lower prevalence ${ }^{11}$. Since the recent data for Sri Lanka is suggestive of a prevalence $<40 \%$ the smaller dose of $30 \mathrm{mg}$ should be adequate for Sri Lanka. Smaller doses have the benefit of minimising the unwanted side effects of higher doses of iron. These recommendations are aimed at treating women with varying degrees of latent ID, as well as to prevent women developing IDA during the pregnancy. This is a low cost alternative to assessment of SF levels to identify high-risk groups.

Antenatal oral iron supplementation should be commenced as soon as the gastro intestinal side effects of early pregnancy have decreased (eg. 12 weeks gestation) and combined with folic acid supplementation $(400 \mu \mathrm{g}$ per day $)^{11}$. In settings where the estimated prevalence of anaemia is $<20 \%$, a weekly dose of $120 \mathrm{mg}$ elemental iron and $2.8 \mathrm{mg}$ of folic acid is recommended for non anaemic pregnant women ${ }^{17}$. In all these situations if a pregnant woman is found to be anaemic after commencement of supplementation, higher / therapeutic doses of iron should be administered.

Many women develop IDA in spite of the well established iron supplementation programs. The low effectiveness of these programs has been attributed to lack of compliance which is mainly due to the troublesome gastro intestinal side effects, improper storage of tablets compromising their pharmacokinetics, poor motivation and lack of awareness of their value, dietary habits and inappropriate methods of taking the oral iron supplements ${ }^{18-20}$. Simple educational interventions can improve the effectiveness of the antenatal oral iron supplementation programs ${ }^{21}$. If gastro intestinal side effects occur, the dose of elemental iron should be reduced. The addition of anthelminthic medications in appropriately selected populations has also been shown to improve the effectiveness of antenatal oral iron supplementation ${ }^{22}$.

The benefits of iron supplementation in pregnancy extend well beyond the period of pregnancy. It has been associated with improved maternal iron stores in the postpartum period, which is especially important when inter pregnancy intervals are short, as the woman will enter a subsequent pregnancy with better iron status. The 
WHO recommends routine postnatal iron supplementation with the same antenatal oral iron supplementation regimen for at least three months ${ }^{23}$.

As there is evidence of poor pregnancy outcome such as low birth weight and prematurity associated with $\mathrm{Hb}>130 \mathrm{~g} / \mathrm{l}$, and higher doses of oral iron are associated with this haemoconcentration and adverse gastrointestinal effects ${ }^{11}$, lower doses of antenatal oral iron supplements should be administered as far as possible, like in any other therapeutic intervention. However, antenatal supplementation as a direct cause of this haemoconcentration has not been established ${ }^{24}$. There are many other alternative methods of iron supplementation described, such as food fortification and home fortification of food with micronutrient powders, but the most cost effective and effective method should be selected to suit the local population, especially in resource poor settings ${ }^{25}$.

Ideally, in order to obtain good pregnancy outcomes, the woman should be iron replete and not anaemic before she conceives. Therefore in settings where the prevalence of anaemia in non pregnant women of reproductive age is $>20 \%$, the $\mathrm{WHO}$ recommends weekly oral supplements of $60 \mathrm{mg}$ elemental iron and $2.8 \mathrm{mg}$ of folic acid for all menstruating adolescent girls and women (10-49 years) for three months followed by a supplement free interval of three months, alternately. This should be repeated cyclically, throughout the reproductive age ${ }^{17}$. Selecting a fixed day of the week as 'WIFS Day' or 'Iron Day' could address the problem of forgetfulness and compliance ${ }^{26}$. In Sri Lanka the prevalence of anaemia in all non-pregnant women between 1549 years of age, was estimated to be approximately $22.2 \%$ using a sample of 2139 non-pregnant women between 15-49 years of age from nine districts of Sri Lanka². Therefore a weekly oral iron supplementation program has been commenced among schoolgirls of Sri Lanka.

\section{TREATMENT OF DEFICIENCY ANAEMIA}

IRON

Management of IDA in pregnancy should be started in the community without delaying interventions awaiting a specialist clinic appointment. The trial of therapeutic oral iron can be undertaken even at the community setting, and if the $\mathrm{Hb}$ improves, the treatment can be continued longer.

The common oral iron preparations available are ferrous sulphate, ferrous fumarate and ferrous gluconate. The corresponding amounts of $60 \mathrm{mg}$ of elemental iron are $300 \mathrm{mg}$ of ferrous sulphate heptahydrate, $180 \mathrm{mg}$ of ferrous fumarate and $500 \mathrm{mg}$ of ferrous gluconate. The dose required for treatment of IDA is $100-200 \mathrm{mg}$ of elemental iron per day ${ }^{6}$. Higher doses should not be used as the iron absorption is saturated at this level and further iron will only increase the gastrointestinal side effects.

Proper advice on taking iron tablets should be an integral part of prescription. The antenatal oral iron supplements should be ideally taken at least one hour before a main meal, together with vitamin $\mathrm{C}$ or some fruit juice, but not with tea or coffee, as polyphenols found in these inhibit iron absorption. The antenatal calcium supplement should be taken at a different time as calcium inhibits non-haeme as well as haeme iron absorption. Consumption of food containing haeme iron, such as red meat, poultry and fish should be encouraged, according to their dietary preferences. Plain cereal or legumebased meals, which do not contain enhancers of iron absorption, should be avoided due to the inhibitory effects of phytates on iron absorption. Food processing and preparation techniques, such as soaking, germination, and fermentation, can remove or degrade phytate to a varying extent, and therefore should be taken at a separate time of day. Other medication such as proton pump inhibitors can affect iron absorption and such medications should be reviewed ${ }^{27}$. Health education $^{21}$ and other methods such as sending electronic text reminders ${ }^{28}$ have also shown to be effective in improving the effectiveness of supplementation programs and such interventions should be incorporated in to the national programs, where feasible. If a woman experiences troublesome gastrointestinal side effects as a result of iron treatment, the dose should be reduced or a different preparation of iron should be tried. Since oral iron is mainly absorbed in the duodenum, preparations such as enteric-coated tablets, which impair absorption, should not be used. Any woman treated for IDA should receive therapeutic doses of iron for at least three months after a normal $\mathrm{Hb}$ is achieved, in order to replenish the iron stores. If she is still pregnant at this point, she may be changed over to supplementation doses for the rest of the pregnancy.

Parenteral iron is an alternative to oral iron in treatment of IDA. However, due to its higher costs and risks of complications, its use has been limited to selected patients. The indications for parenteral iron therapy include non-compliance or intolerance to oral iron or malabsoption syndromes. Although parenteral iron has the ability to replenish the depleted iron stores more rapidly, the rate of improving the $\mathrm{Hb}$ status is similar to that with oral iron. However, parenteral iron is preferred in patients with IDA in advanced pregnancy closer to the delivery, since it is more effective due to lack of any effect from poor compliance. Since overtreatment with iron can cause harm by toxicity, ID should be confirmed prior to parenteral iron therapy and the correct dose calculated, considering the patient's body weight and the iron deficit. Chronic liver disease and on going systemic infections are considered contraindications and parenteral iron should be avoided in the first trimester. Reactions to the drug including anaphylaxis are potential risks of treatment and appropriate monitoring should be undertaken and facilities to manage such emergencies should be available at places of administration. Different preparations are available including Iron sucrose complex, Iron hydroxide dextran complex, Iron isomaltoside 
and Iron carboxymaltose. However the relative efficacy of these drugs have not been compared. Newer preparations have the advantage of being able to be administered as a single dose in a short time interval and have proven to be safe with a lesser risk of anaphylaxis $(<1: 10,000)^{6,29}$. Parenteral iron can also be used in the treatment of postpartum anaemia, and such treatment has proven to reduce the need for postpartum blood transfusion, which is a more costly intervention with higher risks ${ }^{30}$.

There are very few indications for blood transfusion in treatment of IDA in modern obstetric practice. Such treatment is considered in severe degrees of anaemia $(\mathrm{a} \mathrm{Hb}<70$ $\mathrm{g} / \mathrm{l})$, or when there are signs of decompensation. However, its place in life threatening emergency situations is undisputed. Any woman treated with blood transfusion for severe anaemia in the antenatal period should receive therapeutic doses of iron after the blood transfusion to meet the demands of excessive erythropoiesis that will ensue following destruction of transfused blood cells. Blood transfusion is often considered in the postpartum period to treat anaemia resulting from excessive blood loss at time of delivery. If there is no active bleeding and symptoms of cardiac compromise, other treatment modalities such as parenteral iron treatment need to be considered.

\section{CONCLUSIONS}

The prevalence of IDA in pregnant women in Sri Lanka has probably decreased in recent times but it still remains an important issue, which needs to be addressed. Iron supplementation has proven to be a useful intervention in prevention, but we need to revisit our national program to decide the required doses and the duration. Treatment of IDA can be commenced in the community thus avoiding delays, and parenteral iron should be used when indicated as such interventions reduce the need for blood transfusions. Appropriate screening and treatment is more important in high-risk groups. Latent iron deficiency is emerging as an important clinical entity that warrants treatment and further research should be undertaken in our local population to assess the disease burden and the risks to the mother and her baby.

\section{REFERENCES}

1. WHO. Worldwide prevalence of anaemia 1993 - 2005. WHO Global Database on Anaemia. de Benoist B, Mclean E, Egli I, Cogswell M. Eds. Geneva, World Health Organization, 2008 (NLM: WH 155).

2. Jayatissa R, Hossaine SMM. Nutrition and Food Security Assessment in Sri Lanka 2009. Medical Research Institute, Sri Lanka 2010.

3. Chathurani U, Dharshika I, Galgamuwa $\mathrm{D}$, et al. Anaemia in pregnancy in the district of Anuradhapura, Sri Lankaneed for updating prevalence data and screening strategies. Ceylon Med J 2012; 57:101-6.

4. Prathapan $S$, Lindmark $G$, Fonseka $P$, et al. How good is the quality of antenatal care in the Colombo district of Sri Lanka in diagnosing and treating anaemia? Qual Prim Care 2011; 19:245-50.

5. Haemoglobin concentrations for the diagnosis of anaemia and assessment of severity. Vitamin and Mineral Nutrition Information System. Geneva, World Health Organization, 2011(WHO/NMH/ NHD/MNM/11.1). (http://www.who. int/vmnis/indicators/haemoglobin.pdf, accessed on 07 October 2014).

6. Pavord S, Myers B, Robinson S, et al. UK guidelines on the management of iron deficiency in pregnancy. $\mathrm{Br} \mathrm{J}$ Haematol 2012; 156:588-600.

7. Chanarin I, McFadyen IR, Kyle R. The physiological macrocytosis of pregnancy. Br J Obstet Gynaecol 1977; 84 (7): 504 8.

8. Abbassi-Ghanavati M, Greer LG, Cunningham FG. Pregnancy and laboratory studies: A reference table for clinicians. Obstet Gynaecol 2009; 114(6): $1326-31$.

9. WHO. Iron deficiency anaemia. Assessment, prevention and control: A guide for programme managers. Geneva: World Health Organization, 2001: 132 (WHO/ NHD/01.3).

10. Goonewardene M, Seekkuge J, Liyanage
C. Iron stores and its correlation to haemoglobin levels in pregnant women attending an antenatal clinic. Ceylon Med J 1995; 40:67-9.

11. WHO. Guideline: Daily iron and folic acid supplementation in pregnant women. Geneva, World Health Organization, 2012.

12. Brabin BJ, Hakimi M, Palletier D. An analysis of anaemia and pregnancy related maternal mortality. J Nutr 2001; 131(25 -2) 604 S - 615 S; discussion 614 S-615 S.

13. Khan KS, Wojdyla D, Say L et al. WHO analysis of causes of maternal deaths: a systematic review. Lancet 2006; 367(9516): 1066 -1074.

14. Lozoff B, Beard J, Connor J et al. Long -lasting neural and behavioral effects of iron deficiency in infancy. Nutr Rev 2006; 64 (5 Pt 2): S 34 - S 43

15. Lukowski AF, Koss M, Burden MJ et al. Iron deficiency in infancy and neurocognitive functioning at 19 years: evidence of long - term deficits in executive function and recognition memory. Nutr Neurosci 2010; 13(2): 54 -70 .

16. Everett $L$, Wainwright $S$, Ciantar E. PMM. 21 Antenatal Haemoglobin Levels and Blood Transfusion. Arch Dis Child Fetal Neonatal Ed 2014; 99Suppl 1:A130.

17. WHO. Guideline: Intermittent iron and folic acid supplementation in menstruating women. Geneva, World Health Organization, 2011.

18. Habib F, Albadin EHZ, Alenazy M, Nooh R. Compliance to iron supplementation during pregnancy. J Obstet Gynaecol 2009; 29(6): 487 - 492.

19. Goonewardene IMR, Liyanage C. Efficacy of the current antenatal oral iron supplementation programme. Sri Lanka J Obstet Gynaecol 1996; 18: 14 $-17$.

20. Gillooly M, Bothwell TH, Torrance JD, et al. The effects of organic acids, phytates and polyphenols on the absorption of iron from vegetables. Brit J Nutr 1983; 49: $331-342$.

21. Senanayake HM, Premaratne SP, Palihawadana T, et al. Simple educational intervention will improve the efficacy of routine antenatal iron supplementation. $J$ Obstet Gynaecol Res 2010; 36:646-50. 
22. Atukorala TM, de Silva LD, Dechering $\mathrm{WH}$, et al. Evaluation of effectiveness of iron-folate supplementation and anthelminthic therapy against anemia in pregnancy-a study in the plantation sector of Sri Lanka. Am J ClinNutr 1994; 60:286-92.

23. WHO. Recommendations on: Postnatal care of the mother and newborn. Geneva, World Health Organization, 2013

24. Arija V, Ribot B, Aranda N. Prevalence of iron deficiency states and risk of haemoconcentration during pregnancy according to initial iron stores and iron supplementation. Public Health Nutr 2013; 16:1371-8.
25. Pasricha S-R, Drakesmith $\mathrm{H}$, Black J, et al. Control of iron deficiency anemia in low- and middle-income countries. Blood 2013; 121:2607-17.

26. WHO. Weekly iron folic acid supplementation (WIFS) program for women of reproductive age (WRA): an analysis of best program practices. Geneva, World Health Organization, 2011.

27. Ajmera AV, Shastri GS, Gajera MJ, et al. Suboptimal response to ferrous sulfate in iron-deficient patients taking omeprazole. Am J Ther 2012; 19:185-9.
28. Khorshid MR, Afshari P, Abedi P. The effect of SMS messaging on the compliance with iron supplementation among pregnant women in Iran: a randomized controlled trial. J Telemed Telecare 2014; 20:201-6.

29. Froessler B, Collingwood J, Hodyl NA, et al. Intravenous ferric carboxymaltose for anaemia in pregnancy. BMC Pregnancy Childbirth 2014; 14:115.

30. Broche DE, Gay C, Armand-Branger S, et al. Severe anaemia in the immediate post-partum period. Clinical practice and value of intravenous iron. Eur $\mathrm{J}$ of Obst and Gyne 2005; 123(Suppl 2):S21-7. 